\title{
Präkonzeptionelle Risikoberatung
}

Maren Goeckenjan

8.1 Präkonzeptionelle Beratung als gesundheitsfördernde Maßnahme - 54

8.2 Präkonzeptionelle Beratung zu Einzelaspekten in Bezug auf das Abortrisiko - 56

8.3 Präkonzeptionelle Risikoberatung nach wiederholten Fehl-, Früh- und Totgeburten - 60

Literatur - 62 


\subsection{Präkonzeptionelle Beratung als gesundheitsfördernde Maßnahme}

Grundsätzlich sollten alle Frauen im reproduktiven Alter präkonzeptionell zu individuellen Gesundheitsrisiken in Bezug auf Schwangerschaft, Geburt und Stillzeit ärztlich beraten werden. Durch Bewusstwerden von Risiken können Paare ihr Gesundheitsverhalten bereits vor der Konzeption verändern. Medizinische Voruntersuchungen einschließlich der Optimierung der Einnahme von Mikronährstoffen und von Medikamenten können so vorsorglich im zeitlichen Abstand zum Schwangerschaftseintritt veranlasst werden. Besondere Aspekte der präkonzeptionellen Beratung und ihre spezifische Bedeutung bezüglich des Risikos für Fehl-, Früh- und Totgeburten sind in - Tab. 8.1 dargestellt.

Die Beratung kann in gynäkologischen und allgemeinmedizinischen Praxen erfolgen. Allerdings ist sie in Deutschland bislang für Frauen ohne Gesundheitsrisiken nicht als Gesundheitsleistung anerkannt und kann somit nicht angemessen abgerechnet werden.

(7) Die Schwangerenvorsorge setzt für viele Aspekte der Gesundheitsförderung und Prävention von Risiken für Mutter und Kind zu spät an.

Das Leben eines Kindes wird nachweislich schon durch die Bedingungen in der Pränatalzeit geprägt. Ernährungszustand, Lebensweisen, Wohn- und Arbeitsbedingungen der Schwangeren haben einen weitreichenden Einfluss auf die Entwicklung des Feten und des Kindes nach der Geburt - und im Sinne des "fetal programming" auf das gesamte weitere Leben des Kindes ( $\triangleright$ Kap. 4). Präventionsmaßnahmen gelten dann als effektiv, wenn sie in einer Lebenssituation begonnen werden, die zu einer hohen Motivation für Änderungen führt, dem sog. „teachable moment“. Dies trifft in besonderer Weise vor einer geplanten Schwangerschaft zu, insbesondere aber bei Frauen nach unglücklichem Schwangerschaftsausgang und mit dem Bedürfnis nach gezielter Planung einer erneuten Schwangerschaft.
Im Folgenden werden wichtige Aspekte der präkonzeptionellen Beratung mit Einfluss auf die Fehl-, Früh- und Totgeburtenrate besprochen.

Eine Initiative des Center for Disease Control (CDC) in den USA verankerte 2006 die präkonzeptionelle Beratung stärker in der versorgenden Medizinstruktur. Im Zuge dieser Initiative wurden zentrale Ziele der Gesundheitsförderung bei Frauen vor einer Schwangerschaft definiert (Johnson et al. 2006):

\section{Zentrale Ziele der Gesundheitsförderung}

bei Frauen vor einer Schwangerschaft

- Übernahme der individuellen Verantwortung für die eigene Gesundheit im gesamten Lebensverlauf

- Schaffen von Bewusstsein für die eigene Gesundheit und veränderbare Faktoren

- Vermittlung der Wichtigkeit von Vorsorgeterminen und Einhalten dieser Termine

- Angebot von Interventionen bei erkannten Risiken

- Beratung zwischen Schwangerschaften („interkonzeptionelle Beratung“)

- "Check-up" vor einer geplanten Schwangerschaft

- Bezahlung der präventiven Maßnahmen durch die Gesundheitskassen bei sozial schwächeren Frauen mit niedrigem Einkommen

- Programme der Versorgungsforschung und im Bereich „public health“

- Verbesserung der Kontrolle und Beurteilung von präkonzeptioneller Gesundheit und Gesundheit in der Schwangerschaft

- Etablierung eines wissenschaftlichen Instrumentes, um Risiken vor und in der Schwangerschaft zu monitorieren („pregnancy risk assessment monitoring system", PRAMS)

Schon seit Ende der 80er-Jahre existiert in den USA ein System, mit welchem verlässliche Daten zur Lebenssituation von Schwangeren erhoben werden 
- Tab. 8.1 Bestandteile der präkonzeptionellen Beratung und ihre Bedeutung bei Frauen nach Fehl- und Frühgeburten (nach Farahi u. Zolotor 2013)

\begin{tabular}{|c|c|c|c|}
\hline $\begin{array}{l}\text { Bestandteile der } \\
\text { präkonzeptionellen } \\
\text { Beratung }\end{array}$ & Inhalte und Ziele & $\begin{array}{l}\text { Besondere Aspekte mit } \\
\text { Grad der Evidenz (SORT) }\end{array}$ & $\begin{array}{l}\text { Bedeutung bei } \\
\text { Frauen nach } \\
\text { Fehl-, Früh- oder } \\
\text { Totgeburten }\end{array}$ \\
\hline $\begin{array}{l}\text { Maßnahmen der } \\
\text { Gesundheitsförderung }\end{array}$ & $\begin{array}{l}\text { Einnahme von Nahrungsergänzungs- } \\
\text { stoffen, insbesondere Folsäure, Optimie- } \\
\text { rung des BMI }\left(<35 \mathrm{~kg} / \mathrm{m}^{2}\right) \text {, Berücksich- } \\
\text { tigung der sozialen und partnerschaft- } \\
\text { lichen Lebens- und Arbeitssituation }\end{array}$ & $\begin{array}{l}\text { Folsäure (A) } \\
\text { Gewichtsoptimierung (C) }\end{array}$ & ++ \\
\hline Impfstatus & $\begin{array}{l}\text { Nachimpfen bei fehlendem Impfschutz, } \\
\text { Vermeidung von Infektionen }\end{array}$ & $\begin{array}{l}\text { Impfstatus für Masern/ } \\
\text { Mumps/Röteln, Hepatitis, } \\
\text { Varizellen (C) }\end{array}$ & + \\
\hline Sucht & $\begin{array}{l}\text { Begleitung zur Entwöhnung, } \\
\text { Beendigung des Suchtverhaltens }\end{array}$ & Interventionen (B) & ++ \\
\hline $\begin{array}{l}\text { individuelle } \\
\text { medizinische Risiken }\end{array}$ & $\begin{array}{l}\text { Anamnese, Abschätzung von familiären } \\
\text { Erkrankungen, Thromboseneigung, Er- } \\
\text { kennen von internistischen Erkrankun- } \\
\text { gen, Optimierung der Medikation vor } \\
\text { Schwangerschaftseintritt }\end{array}$ & Diabetes mellitus (A) & +++ \\
\hline $\begin{array}{l}\text { geburtshilfliche } \\
\text { Anamnese }\end{array}$ & $\begin{array}{l}\text { Abschätzen des individuellen Risikos } \\
\text { erneuter Komplikationen und ggfs. prä- } \\
\text { ventive Maßnahmen }\end{array}$ & $\begin{array}{l}\text { rezidividierende Aborte } \\
(\mathrm{A}-\mathrm{C})\end{array}$ & +++ \\
\hline $\begin{array}{l}\text { soziale und } \\
\text { psychische Risiken }\end{array}$ & $\begin{array}{l}\text { Abfragen von Faktoren der } \\
\text { Belastungen im Alltag und in der } \\
\text { Paarbeziehung, Erkennen von } \\
\text { psychischen Erkrankungen und } \\
\text { Risiken, Etablieren eines Unterstüt- } \\
\text { zungsplans und konkrete Planung von } \\
\text { Schwangerschaft, Geburt und Stillzeit }\end{array}$ & $\begin{array}{l}\text { Gewalterfahrungen, } \\
\text { soziale Belastungen, } \\
\text { Depression } \\
\text { (C) }\end{array}$ & + \\
\hline
\end{tabular}

A = verlässliche Evidenz guter Qualität, B = inkonsistente oder limitierte Studienaussagen, $C$ = Konsensus, übliche Praxis, Expertenmeinung, Fallserien

können (PRAMS). Dabei werden nicht nur Schwangerschaftsverläufe erfasst, sondern auch soziale Faktoren bis hin zur Erfahrung von Gewalt sowie Vorerkrankungen, bisheriges Kontrazeptionsverhalten, Sucht und Folsäurenutzung mit dem Outcome der Schwangerschaft korreliert. Anhand dieser Datenbanken können aktuelle Fragestellungen gezielt beantwortet werden.

So zeigte z.B. eine Auswertung in einigen Staaten der USA, dass bis 2011 weiterhin relativ wenige Frauen das Angebot einer präkonzeptionellen
Beratung nutzten (32\%). Gerade Frauen mit einem ungeplanten Schwangerschaftseintritt und fehlender Versicherung nahmen dieses Angebot nicht wahr (Williams et al. 2011). Weil Frauen, die in schwierigen sozialen Verhältnissen leben, eher ungeplante Schwangerschaften haben, seltener Vorsorgeuntersuchungen einhalten und insgesamt höhere Risiken für den Schwangerschaftsverlauf aufweisen, wäre es gesundheitspolitisch besonders sinnvoll, eine regelmäßige Beratung vor einem Schwangerschaftseintritt zu etablieren. 


\subsection{Präkonzeptionelle Beratung zu Einzelaspekten in Bezug auf das Abortrisiko}

\subsubsection{Präkonzeptionelle Beratung zu Nahrungsergänzungsstoffen und Vitaminsubstitution}

Die rechtzeitige Folsäuresubstitution vor und in der Schwangerschaft ist eine der bekanntesten präkonzeptionellen Maßnahmen zur Risikoprävention von fetalen Erkrankungen. Die Senkung der Neuralrohrdefekte durch präventive Einnahme von Folsäure ist mehrfach belegt (DeRegil et al. 2010). Die vulnerable Phase für die Entstehung von Fehlbildungen sind die ersten Schwangerschaftswochen. Fehlgeburten können aufgrund unentdeckter schwerwiegender Fehlbildungen des Kindes auftreten (Byrne u. Warburton 1986), so dass vermutet werden kann, dass eine ausreichende präkonzeptionelle Versorgung mit Folsäure auch zu einer Senkung der Fehlgeburtenrate führt. Zusätzlich wird bei Folsäuremangel von Veränderungen der Zellteilung, Entzündungsreaktionen und vermehrtem oxidativen Stress ausgegangen (Byrne 2011). Der Effekt zur Senkung von Fehlgeburten durch Folsäuresubstitution ist jedoch nicht eindeutig belegt. In einer aktuell ausgewerteten Befragungs von Frauen nach Schwangerschaften wurden Selbstangaben zur Folsäuresubstitution und der Schwangerschaftsverlauf untersucht. Der Vergleich von Frauen mit und ohne Folsäuresubstitution ergab eine signifikante Minimierung von geburtshilflichen Risiken bei höherer Folsäureeinnahme über $730 \mu \mathrm{g} / \mathrm{die}$ im Vergleich zu fehlender oder geringerer Einnahme (Gaskins et al. 2015).

Eine ausreichende Datenlage bezüglich einer möglichen Senkung der Frühgeburtsrate durch eine präkonzeptionelle Folsäuresubstitution liegt ebenfalls nicht vor. So fand eine prospektive Kohortenstudie aus Norwegen, bei der mehr als 66.000 Frauen untersucht wurden, keinen protektiven Effekt für eine Frühgeburt durch eine präkonzeptionelle Folsäuresubstitution und Vitamineinnahme (Sengpiel et al. 2014). In einer aktuellen Auswertung der USamerikanischen Risikoüberwachung für Schwangerschaftsrisiken (PRAMS) zeigt sich, dass trotz bestehender Empfehlungen nur 30\% der Frauen im Vormonat vor Schwangerschaftseintritt täglich Folsäure eingenommen hatten (Bixenstine et al. 2015). Besonders bei jungen und nicht-verheirateten Frauen scheint der wiederholte Hinweis auf die regelmäßige präkonzeptionelle Einnahme von Folsäure nötig.

Nahrungsergänzungsstoffe und Vitamine werden heute in Deutschland Frauen mit Kinderwunsch und in der Schwangerschaft großzügig empfohlen. Jodid, Eisen- und Vitamin-D-Substitution erscheinen grundsätzlich vor und in Schwangerschaften sinnvoll, Studien zur Senkung des Abortrisikos liegen jedoch nicht vor. Ob und wann die Substitution präkonzeptionell begonnen werden sollte, ist unklar. Metaanalysen zur Substitution von Vitamin $\mathrm{C}$ und $\mathrm{E}$ in der Schwangerschaft finden keine wissenschaftliche Evidenz in Bezug auf die Senkung von Schwangerschaftskomplikationen (Rumbold et al. 2015), daher ist zu vermuten, dass auch die präkonzeptionelle Gabe keinen präventiven Effekt bewirkt.

Auch wenn die Daten zur Risikominimierung nicht eindeutig sind, lässt sich zeigen, dass nach präkonzeptioneller Beratung nachweislich der Anteil von Frauen steigt, die Nahrungsergänzungsstoffe nutzen und möglicherweise in der Schwangerschaft mit Gesundheitsaspekten bewusster umgehen (Williams et al. 2011).

\subsubsection{Präkonzeptionelle Beratung zur Gewichtsoptimierung}

Ernährungsempfehlungen können langfristig verschiedene Gesundheitsaspekte verbessern. Besonders das Risiko, eine arterielle Hypertonie bzw. Diabetes zu entwickeln, kann mit einer Optimierung des zuvor erhöhten BMI gesenkt werden. Die beste Empfehlung ist die Integration von ausgewogener Ernährung und regelmäßiger Bewegung in den Alltag. 
Sowohl mütterliches Unter- als auch Übergewicht bis hin zur Adipositas haben Folgen für die Fertilität und den Verlauf einer Schwangerschaft (Dean et al. 2014). Besonders klar sind die Zusammenhänge zwischen mütterlichem Untergewicht und niedrigem Geburtsgewicht mit einer RR von 1,64 (95\% CI 1.22-2.21) bzw. Frühgeburt mit einer RR von 1,32 (95\% CI 1.22-1.43). Für Übergewicht und Adipositas ist eine klare Assoziation mit Gestationsdiabetes, Frühgeburt, vaginal-operativen Entbindungen und „fetal distress“ während der Geburt nachgewiesen.

Erfolgen die Maßnahmen zur Reduktion des Gewichtes erst nach Eintritt der Schwangerschaft, sind keine Verbesserungen des Schwangerschaftsverlaufs zu erreichen (Ronnberg et Nilsson. 2010). Hieraus ergibt sich die Notwendigkeit der Gewichtsoptimierung vor Eintritt der Schwangerschaft. Nur präkonzeptionell ist es beispielsweise möglich, bei extremer Adipositas eine chirurgische Intervention zu planen, die in Metaanalysen zu einer signifikanten Senkung von Gestationsdiabetes und Makrosomie des Kindes führte (ACOG Commitee 2013).

\subsubsection{Präkonzeptionelle Beratung zu sozialen und familiären Belastungen}

Psychische, soziale und familiäre Belastungssituationen haben einen Einfluss auf Schwangerschaftsverlauf und -risiken. Auch wenn es schwer ist, bei der multifaktoriellen Genese von Komplikationen einzelne soziale Faktoren als Risiko zu identifizieren, ist es sinnvoll, in der präkonzeptionellen Beratung soziale Belastungen im Alltag zu erfragen. Mögliche Hilfsmittel zur Abschätzung und Beratung von Frauen bezüglich sozialer Risikofaktoren sind einfache offene Fragen, mit denen die finanzielle Situation, Wohnverhältnisse, familiäre Konflikte, Partnerschaftskonflikte und Gewalterfahrungen erfasst werden können (Goeckenjan et al. 2009):

\section{Praxistipp}

Screeningfragen zur sozialen und familiären Situation von Frauen

1. Fühlen Sie sich häufig allein?

2. Wünscht sich Ihr Partner Kinder?

3. Haben Sie im Alltag ausreichende Unterstützung?

4. Gibt es Gründe, die Sie davon abhalten, zu medizinischen Untersuchungen zu kommen, z.B. Schwierigkeiten am Arbeitsplatz oder Schwierigkeiten den Transport betreffend?

5. Fühlen Sie sich an Ihrem Wohnort bedroht?

6. Wurden Sie im letzten Jahr körperlich bedroht, geschlagen, gestoßen oder getreten?

7. Hat Sie jemand zu sexuellem Kontakt, den Sie nicht wollten, gezwungen?

8. Wie hoch geben Sie auf einer Skala von 1 (niedrig) bis 5 (hoch) Ihre Stressbelastung an?

9. Wie häufig sind Sie in den letzten 12 Monaten umgezogen?

10. Wenn Sie die Schwangerschaft nochmal rückgängig machen könnten, würden Sie die Schwangerschaft lieber ... - früher gehabt haben? - auf später verschieben? gar nicht wünschen? - nicht ändern wollen?

Falls die betroffene Frau bei bestimmten Aspekten der Lebenssituation Bedarf an mehr Beratung hat, können Familienberatungsstellen auch schon vor einer Schwangerschaft erste Anlaufadressen sein.

\subsubsection{Präkonzeptionelle Beratung zu Sucht}

Gesundheitsschädigende Lebensgewohnheiten wie Nikotin-, Alkohol-, Drogen- bzw. MedikamentenAbusus haben einen nachgewiesenen negativen Einfluss auf die Fertilität und den Verlauf von Schwangerschaft, Geburt und das spätere Leben der Kinder. 
Jede Schwangere - unabhängig von ihrer sozialen Position, Beruf und Ausbildung - sollte zu diesen Lebensgewohnheiten befragt werden. Zahlreiche Studien konnten eine Risikoerhöhung für Fehl- und Frühgeburten durch Nikotin, Alkohol- und Drogenkonsum zeigen (Blanco-Munoz et al. 2009).

Besteht eine tragfähige Beziehung zwischen Arzt bzw. Ärztin und Patientinnen, können auch tabuisierte gesundheitsschädigende Aspekte angesprochen werden. Die alleinige Beratung bezüglich Suchtverhalten mit dem Hinweis der Suchtvermeidung scheint jedoch nicht zu nachweisbaren Verhaltensänderungen zu führen (Oza-Frank et al. 2015). Daher ist es wichtig, dass nach Kenntnis des Suchtverhaltens verschiedene Unterstützungs- und Interventionsangebote gemacht werden. Kurzinterventionen zur Vermeidung von alkoholbedingten fetalen Entwicklungsstörungen und Schwangerschaftskomplikationen sind effektiv und sollten genutzt werden (Floyd et al. 2007).

\subsubsection{Präkonzeptionelle Beratung zu Impfungen}

Es gibt eine Vielzahl von Infektionen mit potenziell schädigender Wirkung auf eine Schwangerschaft. Dazu gehören insbesondere Röteln, Varizellen, Masern, Pertussis und Influenza. Gegen diese Erkrankungen sollte durch Schutzimpfungen bereits vor einer Schwangerschaft eine Impfimmunität erreicht werden, so dass die Erkrankungen bei Erregerkontakt nicht auftreten. Gegen Cytomegalie-, Parvovirus B19 und Ebstein-Barr-Virus sowie Toxoplasmose und den aktuell mit schweren fetalen Fehlbildungen assoziierten Zika-Virus sind aktive Impfungen bislang noch nicht verfügbar. Eine präkonzeptionelle Beratung umfasst die Kontrolle des Antikörperstatus und bei fehlender Immunität Impfungen vor Schwangerschaftseintritt.

Ziele der präkonzeptionellen Impfberatung

- Erhalten des allgemeinen Impfschutzes in der Schwangerschaft, insbesonders hinsichtlich Diphtherie, Pertussis, Tetanus
- Impfschutz bei besonderen Risiken, z.B. Hepatitis B

- Vermeiden von Fehlgeburten durch Infektionskrankheiten wie Masern (Guillet et al. 2013)

- Vermeiden von schweren Verläufen der Infektionskrankheiten mit akuter Beeinträchtigung der Gesundheit der Schwangeren wie bei Virusgrippe, Pneumokokken-Pneumonie, Maserninfektion, Windpocken

- Vermeiden von Frühgeburten als Infektionsfolge

- Vermeiden einer gestörten intrauterinen Entwicklung mit kongenitalen Virussyndromen, besonders bei Röteln und Varizellen, Infektionen mit dem Zika-Virus

- Vermeiden frühzeitiger Erkrankungen des Neugeborenen durch fehlenden Nestschutz, z.B. bei Masern, Pertussis, Varizellen

\subsubsection{Präkonzeptionelle Beratung bei chronischen Erkrankungen der Mutter}

Aufgrund des zunehmenden Alters bei Familiengründung in Deutschland treten bei Schwangeren gehäuft chronische Erkrankungen wie Asthma, arterielle Hypertonie, Diabetes mellitus, Autoimmunerkrankungen und Schilddrüsenfunktionsstörungen auf, die vor Beginn der Schwangerschaft möglichst optimal behandelt werden sollten. Eine individuelle Schwangerschaftsplanung in interdisziplinärer Kooperation ist das Ziel der präkonzeptionellen Beratung bei chronisch kranken Frauen (•Tab. 8.2). Im Rahmen der präkonzeptionellen Beratung sollten weitere Untersuchungen und ggfs. die interdisziplinäre Umstellung der Medikation unter Vermeidung von embryo- oder fetotoxischen Medikamenten erwogen werden.

Besonders chronisch erkrankte Frauen mit Lupus erythematodes (LE) weisen ein erhöhtes Risiko in der Schwangerschaft für frühe Fehlgeburten und vielfältige weitere Schwangerschaftskomplikationen 
- Tab. 8.2 Präkonzeptionelle Beratung bei chronischen Erkrankungen und Medikation in Bezug auf Abortrisiko (Empfehlungen in Anlehnung an Goeckenjan et al. 2013)

\begin{tabular}{|c|c|c|}
\hline Erkrankung & $\begin{array}{l}\text { Präkonzeptionelle Beratung und mögliche } \\
\text { Therapie }\end{array}$ & Risiko für Fehlgeburten \\
\hline $\begin{array}{l}\text { Arterieller } \\
\text { Hypertonus }\end{array}$ & $\begin{array}{l}\text { - Umstellung der Medikation auf für die } \\
\text { Schwangerschaft zugelassene Antihyper- } \\
\text { tensiva } \\
\text { - Hinweis auf Schwangerschaftsrisiken und } \\
\text {-überwachung } \\
\text { - Abklärung der renalen Funktion und } \\
\text { möglicher Begleiterkrankungen }\end{array}$ & $\begin{array}{l}\text { - erhöhtes Thrombose- und Abortrisiko durch } \\
\text { Begleiterkrankungen (LE, DM, aPLS) } \\
\text { - Überwachung der Risikoschwangerschaft } \\
\text { - erweitertes Ersttrimester-Screening }\end{array}$ \\
\hline $\begin{array}{l}\text { Diabetes mellitus } \\
\text { (DM) }\end{array}$ & $\begin{array}{l}\text { präkonzeptionelle Optimierung der Blut- } \\
\text { zuckereinstellung, HbA1c möglichst unter } \\
6,5 \% \text {, präkonzeptionelle Planung der Thera- } \\
\text { pie, gute Überwachung in der Schwanger- } \\
\text { schaft }\end{array}$ & $\begin{array}{l}\text { - Assoziation mit PCOS } \\
\text { - RR für Fehlgeburt bei Diagnose von Typ } \\
2 \text { DM 1,3 und für intrauterinen Fruchttod von } \\
\text { 2,5 - unabhängig von präkonzeptioneller } \\
\text { Einstellung (Jovanovic et al. 2015) }\end{array}$ \\
\hline Depression & $\begin{array}{l}\text { Beratung zu medikamentöser Therapie und } \\
\text { zu Schwangerschaftsverlauf }\end{array}$ & $\begin{array}{l}\text { Hinweise auf leicht erhöhtes Abortrisiko bei } \\
\text { Einnahme von bestimmten Antidepressiva } \\
\text { (z.B. Paroxiten und Venlafaxin) in der Schwan- } \\
\text { gerschaft (Kjaersgaard et al. 2013) }\end{array}$ \\
\hline Epilepsie & $\begin{array}{l}\text { Umstellen der antiepileptischen Therapie, } \\
\text { Kontrolle der Spiegel }\end{array}$ & $\begin{array}{l}\text { erhöhtes Risiko für Fehlgeburten mit einer OR } \\
\text { von 1,5, Frühgeburt OR 1,2 (Viale et al. 2015) }\end{array}$ \\
\hline Hyperthyreose & $\begin{array}{l}\text { Umstellung auf Propylthiouracil, gute Stoff- } \\
\text { wechselkontrollen in der Schwangerschaft, } \\
\text { direkt postnatal erhöhtes Risiko einer } \\
\text { schweren Hypothyreose beim Neonaten }\end{array}$ & $\begin{array}{l}\text { signifikant erhöhtes Abortrisiko bei Hyper- } \\
\text { thyreose mit RR von 1,2-1,3 im Vergleich zu } \\
\text { gesunden Frauen (Andersen et al. 2013) }\end{array}$ \\
\hline Hypothyreose & $\begin{array}{l}\text { zumeist Empfehlung zu TSH-Zielwert prä- } \\
\text { konzeptionell und in der Schwangerschaft } \\
\text { unter 2,5 mU/l, Anpassung der L-Thyroxin- } \\
\text { Dosis an den Bedarf in der Schwangerschaft } \\
\text { (Budenhofer et al. 2013), TSH-Kontrollen alle } \\
3 \text { Monate bei Hashimoto ggfs. Selen }\end{array}$ & $\begin{array}{l}\text { Assoziation von latenter und manifester Hypo- } \\
\text { thyreose mit TSH > } 4 \mathrm{mU} / \mathrm{l} \text { und Abortrisiko } \\
\text { nachgewiesen (ASRM Guideline 2015); ob } \\
\text { diese Assoziation auch für subklinische Hypo- } \\
\text { thyreosen (TSH 2,5-4 mU/l) gilt, ist umstritten; } \\
\text { bei Hashimoto-Thyreoditis jedoch klare Asso- } \\
\text { ziation mit Abortneigung }\end{array}$ \\
\hline Zöliakie & $\begin{array}{l}\text { Optimierung der Diätadhärenz internisti- } \\
\text { sche Kooperation }\end{array}$ & $\begin{array}{l}\text { Risiko für Fehlgeburten bei Zöliakie erhöht } \\
\text { RR 1,4 (Tersigni et al. 2014), sinkt jedoch bei } \\
\text { guter Diät }\end{array}$ \\
\hline
\end{tabular}

auf; so entwickelt beispielsweise mehr als ein Fünftel aller Schwangeren mit LE eine Präeklampsie (RuizIrastorza u. Khamashta 2011). Präkonzeptionell muss das individuelle Gesundheitsrisiko durch eine Schwangerschaft bei einer chronisch kranken Frau abgeschätzt werden. Bei pulmonalem Hypertonus, restriktiver Lungenerkrankung, schwerer Einschränkung der kardialen und pulmonalen Funktion sowie chronischer Niereninsuffizienz sollte jedoch von einer Schwangerschaft abgeraten werden.
Eine zunehmende Erkrankung im reproduktiven Alter ist der Diabetes mellitus Typ 2 (T2DM), häufig in Kombination mit einem polycystischen Ovar-Syndrom (PCOS; Knochenhauer et al. 1998). Für das PCOS ist bekannt, dass ein erhöhtes Abortrisiko besteht, besonders bei Hyperandrogenämie und Insulinresistenz (Cocksedge et al. 2008). Verschiedene präkonzeptionell begonnene Interventionen wie Metformingabe bei Insulinresistenz, Gewichtsreduktion und Senkung der zuvor erhöhten Androgenspiegel können das Abortrisiko senken. 


\subsection{Präkonzeptionelle Risikoberatung nach wiederholten Fehl-, Früh- und Totgeburten}

Insbesondere nach Fehl-, Früh- oder Totgeburten ist eine individuelle Beratung vor Eintritt einer erneuten Schwangerschaft sinnvoll. Diese Beratung birgt Chancen, den zukünftigen Schwangerschaftsverlauf zu verbessern und psychologische und körperliche Belastungen durch weitere, sich manifestierende Risiken zu minimieren.

Im optimalen Fall sollte die Beratung in einer spezialisierten Sprechstunde erfolgen. Vorschläge für spezialisierte Fehlgeburtensprechstunden mit speziell trainiertem Personal und strukturiertem Vorgehen sowie ausreichend Zeit für die Anamnese wurden schon Ende der 90er-Jahre gemacht ( $\mathrm{Li}$ 1998). Eine psychologische Begleitung bei Paaren mit wiederholten Fehlgeburten kann im Rahmen dieser Sprechstunde angeboten und gebahnt werden.

\subsubsection{Psychische Belastungssituation bei Paaren mit Fehlgeburt und wiederholtem Spontanabort (RSA)}

Unterstützungshilfen zur Verarbeitung von Trauer nach Fehlgeburten werden in der gynäkologischen Praxis und besonders verstärkt in der Spezialsprechstunde für RSA angeboten. Nach einer qualitativen Analyse in den Niederlanden zu von den Betroffenen gewünschten Unterstützungsangeboten ergeben sich folgende konkrete Bedürfnisse der Frauen mit Fehlgeburten (Musters et al. 2011):

\section{Bedürfnisse von Frauen mit Fehlgeburten}

- Bedürfnisse während der medizinischen Betreuung:

- möglichst kontinuierliche Betreuung durch eine oder zwei gut ausgebildete Gynäkologen

- enge Beziehung zu Kontaktpersonen auch im Verlauf, Fragen nach Wohlbefinden und emotionalen
Bedürfnissen, Verständnis durch Betreuende

- individuelle Beratung zu Lebensstil, Ernährung und zusätzlichen internetbasierten Beratungsangeboten

- konkrete Feinplanung der ersten zwölf Schwangerschaftswochen in der nächsten Schwangerschaft

- umfassende individuelle und bedürfnisorientierte Betreuung, falls es zu erneuten Fehlgeburten kommt

- Beta-HCG-Verlauf in der frühen Schwangerschaft

- regelmäßige und engmaschige Ultraschalluntersuchungen in der Frühschwangerschaft alle ein bis zwei Wochen und bei Beschwerden

- Beratung zur Medikamentengabe und -wirkung in der Schwangerschaft

- möglichst keine gemeinsamen Wartezimmer für schwangere und nicht-schwangere Frauen

- gewünschte weiterführende Unterstützungsangebote

- Kontakt zu Sozialdienst

- Beratung zu Entspannungsübungen, Sport

- Einbeziehen des Partners in Beratung und Therapie

Frauen mit RSA leiden auch in nachfolgenden Schwangerschaften stärker unter Trauer, Angst und Selbstzweifeln als Frauen ohne Fehlgeburten in der Anamnese (z.B. Fertl et al. 2005). Dass sich auch schon in der Frühschwangerschaft eine Bindung zwischen der Mutter und dem noch ungeborenen Kind entwickelt und durch Fehlgeburten Trauerreaktionen hervorgerufen werden, ist heute anerkannt. Auch die psychische Belastung des Vaters ist seit Ende der 90er-Jahre beschrieben worden (Puddifoot u. Johnson 1997) und wird zunehmend berücksichtigt. Daher sollten die Gespräche zur Aufklärung über mögliche Gründe der Fehlgeburt und Folgen für die weiteren Schwangerschaften möglichst gemeinsam mit dem Paar erfolgen. 
Frauen mit RSA und nach Schwangerschaftskomplikationen wie Früh- und Totgeburt gelten als schwierige Patientinnen. Die Kombination aus Leidensdruck, Verzweiflung und guter Information bedingt oft eine hohe Bereitschaft für eine zusätzliche und kostenintensive Diagnostik und Risikobereitschaft für nicht evidenz-basierte medizinische Maßnahmen. Daher ist die Patientenbindung und -führung in speziellen Sprechstunden sehr zeit- und personalintensiv.

Wie besonders auf die medizinische und psychologische Risikokonstellation von mehrfachen Fehlgeburten in speziellen Sprechstunden eingegangen werden kann, beschreiben Wallerstedt et al. (2003). Folgende grundsätzliche Aspekte sollten in der Fehlgeburtensprechstunde thematisiert werden:

- Wahrnehmung und Anerkennung von Trauer und Angst, Unterstützung der Trauerarbeit Beschreibung der Trauerprozesse und Einordnung in den Verlauf von anderen Paaren in ähnlichen Situationen

- Thematisierung von Hoffnung und Enttäuschung, Ansprechen der Situation der Fehlgeburt, Benennen des Kindes

- Auswirkungen auf Selbstwert und Selbstbewusstsein, Einordnen in die individuelle Biografie

- besondere Aspekten der Paarbeziehung: Kommunikation, Sexualität, Umgehen mit Konflikten

- bereits genutzte und/oder erwünschte Unterstützungsangebote

\subsubsection{Besondere Beratungsaspekte}

Welche Frauen mit anamnestischen Fehlgeburten sollten in speziellen Sprechstunden beraten werden?

Aufgrund der nicht-einheitlichen Definition der Diagnose „wiederholte spontane Aborte“ werden häufig auch schon Frauen mit weniger als drei Fehlgeburten in den Sprechstunden beraten. Es ist sinnvoll, bei diesen Frauen auf den Leidensdruck einzugehen und eine differenzierte, wenngleich abgestuft geringere Abklärung durchzuführen.

Wie lange sollte das Intervall zwischen der Fehlgeburt und dem nächsten Schwangerschaftseintritt sein?

Lange Zeit wurden Frauen nach Fehlgeburten auf eine sinnvolle Verzögerung bis zum nächsten
Schwangerschaftseintritt hingewiesen. Eine rasche Schwangerschaftsfolge nach einer Geburt, definiert als $<18$ Monate bis zum nächsten Schwangerschaftseintritt, führt zu einem signifikant höheren relativen Risiko für Mutter und Kind im Vergleich zu längeren Intervallen (Zhu et al. 1999). Im Gegensatz dazu gibt es keine schlüssigen Studien zum Intervall bis zur nächsten Schwangerschaft nach Fehlgeburten. Viele der Studien wurden in sich entwickelnden Ländern durchgeführt und schlossen auch Schwangerschaftsabbrüche mit hohen Infektionsraten ein (Bhattacharya u. Smith 2011). Dennoch lautete die weiterhin gültige Empfehlung der WHO, nach einer Fehlgeburt mindestens sechs Monate zu warten, bevor eine neue Schwangerschaft eintreten sollte (WHO 2005). Möglicherweise zeigt sich sogar eine Verbesserung des Schwangerschaftsverlaufs bei raschem Eintritt einer Schwangerschaft nach einer Fehlgeburt. Die Analyse von Sekundärergebnissen einer prospektiven Studie zu Acetylsalicylsäure nach Fehlgeburten in der Frühschwangerschaft ergab $(n=1.228)$, dass Frauen mit einem kurzen Intervall von unter drei Monaten bis zum erneuten Schwangerschaftseintritt keine Risikoerhöhung für eine erneute Fehlgeburt, Frühgeburt, Präeklampsie oder Schwangerschaftsdiabetes aufwiesen (Wong et al. 2015).

(7rauen nach Fehlgeburten müssen keine Verzögerung ihres Schwangerschaftswunsches in Kauf nehmen.

Die Diagnostik sollte jedoch vor erneuter Schwangerschaft abgeschlossen und im Hinblick auf mögliche Interventionen ausgewertet sein.

\section{Welche Aspekte sollten bei der präkonzeptionel- len Risikoberatung nach intrauterinem Fruchttod besonders angesprochen werden?}

Der Umgang mit Eltern nach einer Totgeburt, Geburt eines pränatal verstorbenen Kindes oder nach induzierten Aborten hat sich in den letzten 20 Jahren stark geändert. Es wird heute äußerst bewusst mit Trauerreaktionen von Mutter und Vater umgegangen. Erinnerungsstücke werden heute regelhaft an die Eltern ausgegeben und erleichtern den Umgang mit dem Verlust. Es ist bekannt, dass ein Ansprechen des Verlustes und Benennen des Kindes bei späteren Kontakten mit dem Paar die Trauerreaktionen 
erleichtern kann (Moore et al. 2011). Besonders Hebammen und das Personal in der gynäkologischen Praxis können in der Zwischenzeit bis zum Eintritt einer erneuten Schwangerschaft eine Beziehung aufbauen. Das Debriefing nach schwierigen Geburten und die interkonzeptionelle Beratung können dem Paar und besonders der Frau wieder Sicherheit bei der Planung der Folgeschwangerschaft bieten. Bei diesen Risikogesprächen in der gynäkologischen Praxis oder in Risikosprechstunden von Kliniken sollte der Schwerpunkt auf die Ursachen der Früh- oder Totgeburt, die konkrete Schwangerschaftsplanung und die verlässliche Anbindung während der präkonzeptionellen Phase und in der Schwangerschaft gelegt werden. In Abhängigkeit von den Ursachen können verschiedene Maßnahmen bereits präkonzeptionell begonnen werden. Außer der Folsäuresubstitution sind keine weiteren spezifischen Nahrungsergänzungs- und Vitaminsubstitutionen mit einer Risikominimierung für den fetalen und neonatalen Todesfall verbunden (Rumbold et al. 2015).

\section{Literatur}

American College of Obstetricians and Gynecologists (2013) ACOG Committee opinion no. 549: obesity in pregnancy. Obstet Gynecol 121(1): 213-217

Andersen SL, Olsen J, Wu CS, Laurberg P (2014) Spontaneous abortion, stillbirth and hyperthyroidism: a danish population-based study. Eur Thyroid J 3(3): 164-172. doi: 10.1159/000365101

Bhattacharya S, Smith N (2011) Pregnancy following miscarriage: what is the optimum interpregnancy interval? Womens Health 7(2): 139-141. doi: 10.2217/whe.11.2

Bixenstine PJ, Cheng TL, Cheng D, Connor KA, Mistry KB (2015) Association between preconception counseling and folic acid supplementation before pregnancy and reasons for non-use. Matern Child Health J19(9): 1974-1984. doi: 10.1007/s10995-015-1705-2

Blanco-Muñoz J, Torres-Sánchez L, López-Carrillo L (2009) Exposure to maternal and paternal tobacco consumption and risk of spontaneous abortion. Public Health Rep 124(2): 317-322

Budenhofer BK, Ditsch N, Jeschke U et al. (2013) Thyroid (dys-) function in normal and disturbed pregnancy. Arch Gynecol Obstet 287(1): 1-7. doi: 10.1007/s00404-012-2592-z

Byrne J (2011) Periconceptional folic acid prevents miscarriage in Irish families with neural tube defects. Ir J Med Sci 180: 59-62

Byrne J, Warburton D (1986) Neural tube defects in spontaneous abortions. Am J Med Genet 25: 327-333
Caseiro AL, Regalo A, Prereira E et al. (2015) Implication of sperm chromosomal abnormalitities in recurrent abortion and multiple implantation failure. RBM Online doi:10.1016/j.rbmo.2015.07.001

CDC (2006) Recommendations to improve preconception health and health care - United States. A report of the CDC/ATSDR preconception care work group and the select panel on preconception care. MMWR 55(RR06): 1-23

Cocksedge KA, Li TC, Saravelos SH et al. (2008) A reappraisal of the role of polycystic ovary syndrome in recurrent miscarriage. Reprod Biomed Online 17(1): 151-160

Dean SV, Lassi ZS, Imam AM et al. (2014) Preconception care: nutritional risks and interventions. Reprod Health 11: S3

De-Regil LM, Fernández-Gaxiola AC, Dowswell T et al. (2010) Effects and safety of periconceptional folate supplementation for preventing birth defects. Cochrane Database of Systematic Reviews 10: CD00795

Farahi N, Zolotor A (2013) Recommendations for preconception counseling and care. Am Fam Physician 88(8): 499-506

Fertl KI, Bergner A, Beyer R, Klapp BF, Rauchfuss M (2009) Levels and effects of different forms of anxiety during pregnancy after a prior miscarriage. Eur J Obstet Gynecol Reprod Biol 142: 23-29

Floyd RL, Lobell M, Velasquez MM et al. (2007) Preventing alcohol-exposed pregnancies: a randomized controlled trial. Am J Prev Med 32(1): 1-10

Gaskins AJ, Rich-Edwards JW, Hauser R et al. (2014) Maternal prepregnancy folate intake and risk of spontaneous abortion and stillbirth. Obstet Gynecol 124(1): 23-31. doi: 10.1097/AOG.0000000000000343

Goeckenjan M, Holschbach V, Toth B (2014) Präkonzeptionelle Risikoberatung. Gynäkol Endokrinol 12: 35-46

Goeckenjan M, Ramsauer B, Hänel M et al. (2009) Soziales Risiko - geburtshilfliches Risiko? Gynäkologe 42: 102-110

Guillet M, Vauloup-Fellous C, Cordier AG et al. (2012) Measles in pregnancy: a review]. J Gynecol Obstet Biol Reprod 41(3): 209-218. doi: 10.1016/j.jgyn.2012.01.008

Jovanovic L, Liang Y, Wenig W et al. (2015) Trends in the incidence of diabetes, its clinical sequelae, and associated costs in pregnancy. Diabetes Metab Res Rev. doi: 10.1002/ dmrr.2656

Kjaersgaard MI, Parner ET, Vestergaard M et al. (2013) Prenatal antidepressant exposure and risk of spontaneous abortion - a population-based study. PLoS One 8 (8):e72095. doi: 10.1371/journal.pone.0072095

Knochenhauer ES, Key TJ, Kahsar-Miller M et al. (1998) Prevalence of the polycystic ovary syndrome drome in unselected black and white women of the Southeastern United States: A prospective study. J Clin Endocrinol Metab 83: 3078-3082

Leitlinie der DGGG (S1) (2013) Diagnostik und Therapie des wiederholten Spontanaborts. 015/050, Stand: 12/2013

Li TC (1998) Guides for practitioners. Recurrent miscarriage: principles of management. Huma Reprod 13:478-482

Moore T, Parrish H, Black BP (2011) Interconception care for couples after perinatal loss: a comprehensive review of the literature. J Perinat Neonatal Nurs 25(1): 44-51. doi: 10.1097/JPN.0b013e3182071a08 
Musters AM, Taminiau-Bloem EF, van den Boogaard E et al. (2011) Supportive care for women with unexplained recurrent miscarriage: patients' perspective. Human Reprod 26: 873-877

Oza-Frank R, Kachoria R, Keim SA et al. (2015) Provision of specific preconception care messages and associated maternal health behaviors before and during pregnancy. Am J Obstet Gynecol 212(3): 372.e1-8. doi: 10.1016/j. ajog.2014.10.027

Practice Committee of the American Society for Reproductive Medicine (2015) Subclinical hypothyroidism in the infertile female population: a guideline. Fertil Steril 104 (3):545-553. doi: 10.1016/j.fertnstert.2015.05.028

Puddifoot JE, Johnson MP (1997) The legitimacy of griefing: the partner's experience at miscarriage. Soc Sci Med 45(6): 837-845

Ronnberg AK, Nilsson K (2010) Interventions during pregnancy to reduce excessive gestational weight gain: a systematic review assessing current clinical evidence using the Grading of Recommendations, Assessment, Development and Evaluation (GRADE) system. BJOG 117(11): 1327-1334

Ruiz-Irastorza G, Crowther MA, Branch DW, Khamashta MA (2010) Antiphospholipid-syndrome. Lancet 375: 1498-1509

Rumbold A, Ota E, Hori H, Miyazaki C, Crowther CA (2015) Vitamin E supplementation in pregnancy. Cochrane Database Syst Rev 9: CD004069

Rumbold A, Ota E, Nagata C, Shahrook S, Crowther CA (2015) Vitamin C supplementation in pregnancy. Cochrane Database Syst Rev 9: CD004072. doi: 10.1002/14651858. CD004072.pub3

Sengpiel V, Bacelis J, Myhre R et al. (2014) Folic acid supplementation, dietary folate intake during pregnancy and risk for spontaneous preterm delivery: a prospective observational cohort study. BMC Pregnancy Childbirth 14: 375. doi: 10.1186/s12884-014-0375-1

Tersigni C, Castellani R, de Waure C et al. (2014) Celiac disease and reproductive disorders: meta-analysis of epidemiologic associations and potential pathogenic mechanisms. Hum Reprod Update 20(4): 582-593. doi: 10.1093/ humupd/dmu007

Viale L, Allotey J, Cheong-See F et al. (2015) Epilepsy in pregnancy and reproductive outcomes: a systematic review and meta-analysis. Lancet pii: S0140-6736(15)00045-8. doi: 10.1016/S0140-6736(15)00045-8

Wallerstedt C, Lilley M, Baldwin K (2003) Interconceptional Couseling after perinatal and infant loss. JOGNN 32: 533-542. DOI: 10.1177/0884217503255264

Williams L, Zapata LB, D'Angelo DV et al. (2012) Associations between preconception counseling and maternal behaviors before and during pregnancy. Matern Child Health J 16(9):1854-1861. doi: 10.1007/s10995-011-0932-4

Wong LJ, Schliep KC, Silver RM et al. (2015) The effect of a very short interpregnancy interal and pregnancy outcomes following a previous pregnancy loss. Am J Obstet Gynecol 212: 371.e1-11
World Health Organisation (2005) Report of a WHO technical consultation on birth spaching 2005. http://who.int/ maternal_child_adolescent/documents/birth_spacing. pdf (Zugriff: 27.09.2015)

Zhu BP, Rolf RT, Nangle BE, Horan M (1999) Effect of the interval between pregnancies on perinatal outcomes. $\mathrm{N}$ Engl J Med 340: 589 